\title{
Functional Bronchoscopy: Development of a New Bronchoscopic Method for Real-Time Gas Exchange Assessment of Lobes and Lung Segments
}

\author{
Lutz Freitag Dierik Lenkens Paul Zarogoulidis Rüdiger Karpf-Wissel \\ Heiko Hang Kaid Darwiche \\ Department of Interventional Pulmonology, Ruhrlandklinik, University Hospital Essen, University Duisburg-Essen, \\ Essen, Germany
}

\section{Key Words}

Functional bronchoscopy · Endocapnometry - Endoscopic emphysema treatment

\begin{abstract}
Background: There are various imaging methods in use designed to provide information on lung functional status, particularly gas exchange within specific lung segments. These complex imaging methods provide indirect information about volume and local lung function. Objectives: The objective of this research was to develop a simpler and more direct method for the functional assessment of gas exchange in lung segments. Method: We have developed a new bronchoscopic method to sample gas concentrations of oxygen and carbon dioxide at the orifices of segmental bronchi in a breathing patient. Endocapnometry and oximetry curves are displayed in real time and superimposed on the endoscopic video images. Results: The gas exchange mapping of a lung could be achieved in $<5 \mathrm{~min}$. We identified typical curve patterns of localized slow and fast clearance regions, and segments with higher or lower oxygen uptake. This method is simple and versatile and the data displayed can help to identify target zones for endoscopic emphysema treatments, e.g. for lowering the risk of resection surgery, or to improve ventilation strategies in ICU patients. Conclusion: This new method enables gas sampling at the
\end{abstract}

\section{KARGER}

E-Mail karger@karger.com

www.karger.com/res lung segment level. The concomitant display of local endocapnometry and endooximetry curves allows for a better identification of target zones for endoscopic emphysema treatments or to improve ventilation strategies for patients on respiratory support.

(c) 2014 S. Karger AG, Basel

\section{Introduction}

Functional lung imaging methods have become essential tools for planning procedures in thoracic surgery and interventional bronchoscopy. Information regarding the functional status of specific segments of the lung is especially valuable as new treatment modalities for emphysema are being developed, such as valve placement or the application of bio-foam to shut off potentially nonfunctional parenchyma. The most commonly applied imaging method is computed tomography (CT). Using sophisticated densitometry techniques, often combined with time-resolved volumetric calculations, chest radiologists can acquire information about the size of lobes and segments as well as local ventilation and perfusion [1].

These radiographic methods compete with nuclear medicine techniques. Gamma camera images of radioactive gases (e.g. Krypton) or aerosols provide information about ventilation, and radioactive microaggregates in the 
Fig. 1. Functional bronchoscopy with a mass-spectrometer (a) and the new device with built-in gas sensors, catheter flusher, video and storage controller (b).
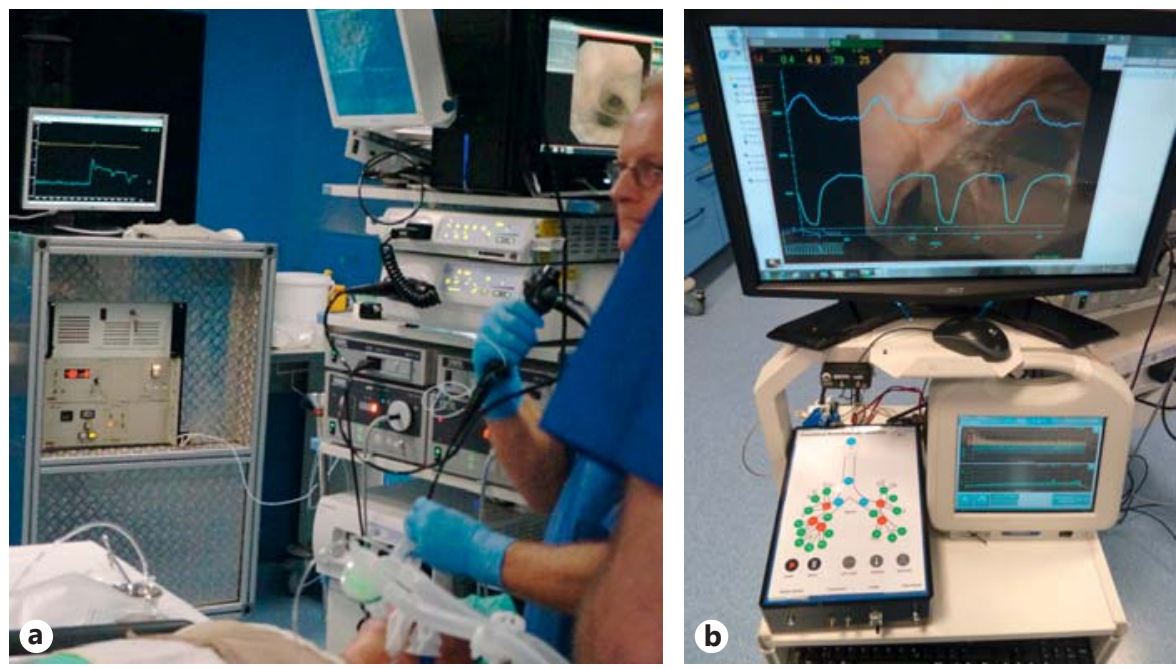

bloodstream are used to visualize perfusion. While ventilation scintigraphy is rarely applied, a Technecium perfusion scan is a standard method in the workup of a patient scheduled for thoracic surgery [2-4]. Magnetic resonance imaging with noble or hyperpolarized gases is technically even more demanding $[5,6]$.

While imaging methods provide indirect information about volumes and local functions, catheter-based techniques have been used for direct measurements of airflows and gas concentrations. Bronchospirometry, made popular in Europe by Fleming and West [7] in 1954, was a typical method for evaluating the functional status of the right and left lungs. Patients were intubated with a Carlens double-lumen tube [8]. Spirometers and, in later developments, gas-analyzers were attached and measurements were performed while postoperative conditions were evaluated [9]. Contemporary images show patients sitting on bicycles, intubated with double-lumen tubes attached to twin-spectrometers. Although Fleming described bronchospirometry as a 'not particularly unpleasant procedure for the patient, ... who views it with considerably more favor than he accords to other more commonly carried out procedures', it was soon replaced by the aforementioned nuclear imaging procedures [10]. Besides its questionable comfort, the bronchospirometry technique with the Carlens tube had a limited regional resolution of ventilation and oxygen uptake in the two lung wings.

Measurements from distal airways became possible when flexible bronchoscopes and smaller catheters became available. Gas sampling methods could be reduced to the lobar and segmental level. Balloon catheters, advanced through the working channel of a bronchoscope, were attached to mass spectrometers. Inert tracer gases were injected and the read-outs of local concentrations were used to estimate the volumes of lobes. Others measured the local concentration of carbon dioxide to estimate the amount a particular lobe contributed to the overall gas exchange [11-15]. One of the most sophisticated techniques was developed in the eighties by Morgan et al. [16]. They used a mass spectrometer attached to a sampling catheter to measure the slope of concentration drops of inhaled Argon and Freon gas mixtures. The dilution of Argon not taken up by the blood provides information about volume and flow, while the slope of highly absorbed Freon is an indicator of local blood perfusion. Although we found this technique feasible, it requires a very large and expensive mass spectrometer, as illustrated in figure 1a.

In the late fifties, Paul Hugh-Jones and John West [17] attached a mass spectrometer to a semirigid sampling catheter and analyzed expiratory carbon dioxide and oxygen curves from the individual lobes of animals. They identified typical curves for arterial and airway obstructions. Again, the instrumental hurdles prevented clinical applicability. Over the years, simple capnometers based on infrared absorption were developed and became broadly applied in anesthesia and emergency medicine. Mass spectrometers were downgraded as research tools and capnometers soon became established as routine instruments for clinical gas analysis. When small capnometers and flexible bronchoscopy eventually became available, they were introduced by Japanese colleagues as components of a minimally invasive procedure for measuring expiratory $\mathrm{CO}_{2}$ at the lobar level $[18,19]$. 
Fig. 2. Endobronchial gas concentrations are collected through a catheter in the working channel of a bronchoscope and superimposed on the actual endoscopic image.

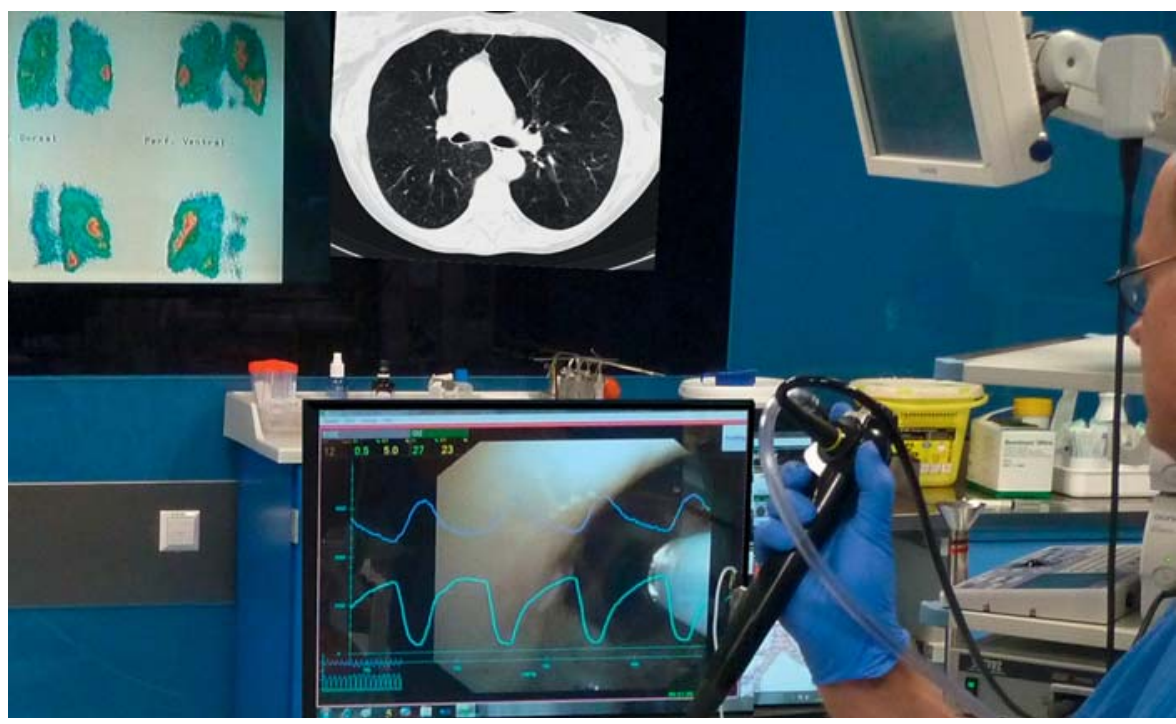

We have taken the idea further and developed an instrument for online analysis and support for decisionmaking in interventional bronchoscopy. The device and technique are described here.

\section{Materials and Methods}

The basis of our device is a pocket-sized capnometer and oximeter with a built in suction pump available as a spare part from an anesthesia machine (Masimo AB, formerly Phasein, Danderyd, Sweden). We added a valve component that is regulated by a microcontroller. Controlled by the bronchoscopist, the analyzer can aspirate either calibration gas (usually room air) or air from a sampling catheter, or else flush the catheter with air from a second pump. The catheter is a regular cytology sampling catheter that is advanced through the working lumen of a bronchoscope. The read-outs from the capnometer and the oximeter are processed by a proprietary custom software program and presented on a monitor visible to the bronchoscopist. The curves for the gas concentrations are displayed in a strip-chart like manner that is superimposed on the video image of the bronchoscope (fig. 2); the time lag due to the length of the catheter is approximately $2 \mathrm{~s}$. When the bronchoscopist moves the tip of the scope from segment to segment, while holding it in position for two breaths, he or she can see the representative signals from that particular segment or lobe almost in real time. A touch pad allows labeling of the images and data (fig. 1b). The bronchoscopic image (video signal) and data from the gas analyzer are stored in the computer for further analysis. If mucus is accidentally aspirated, the catheter is automatically flushed. The system is part of our larger functional bronchoscopy program. We use it for various other measurements, such as airflows, endobronchial pressures, endobronchial blood flows and drug accumulation. The gas sampling module presented here is currently our most sophisticated version. In an ongoing study, we are comparing the data from our functional bronchoscopy adapter with CT images and perfusion scans. 


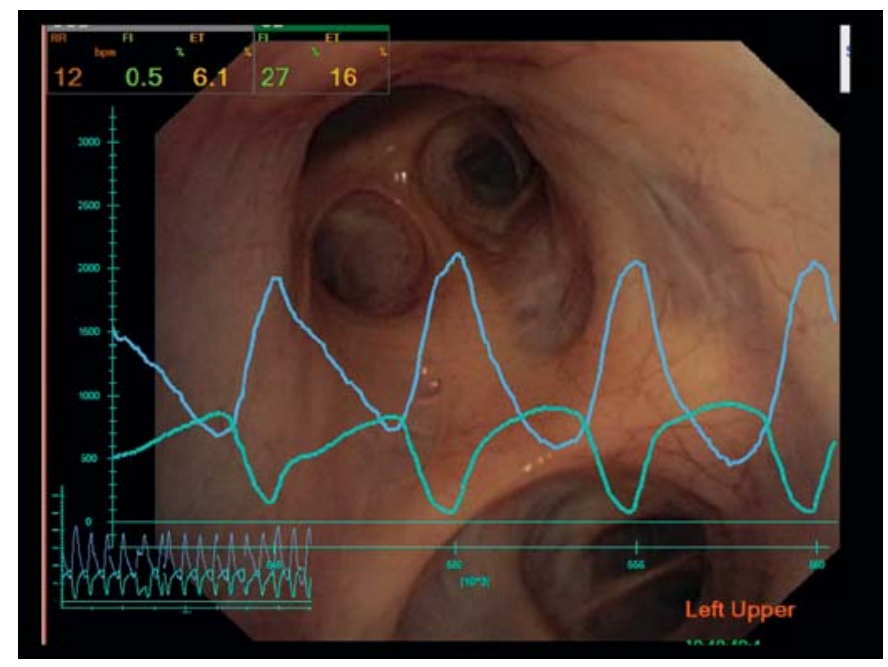

Fig. 4. The catheter is moved (left to right) from a slow space lobe with a triangular $\mathrm{CO}_{2}$ waveform to a region with fast $\mathrm{CO}_{2}$ clearance (lower curve) and high peak-to-trough $\mathrm{O}_{2}$ signal (upper curve).

a triangular shape. In most cases, this is accompanied by smaller amplitude of the oxygen curve (blue, upper curve). The opposite is shown in figure 4 . Here, the catheter is moved from an orifice of an emphysematous destroyed left lower lobe to the upper lobe that shows less destruction on the CT scan. The capnometric curve changes from a triangle to a rectangle shape and the oxygen consumption signal increases.

The changes in shape are not always uniform, as demonstrated in figure 5 . We have seen examples where the capnogram changes without any noticeable modifications in the oxygen curve and vice versa.

Another typical pattern is shown in figure 6. During bronchoscopy, the visible collapse of the airways is accompanied with irregular, often double-hump curves of the gas concentration signals. We always measure collateral ventilation with the Chartis system (Pulmonx Inc., Redwood City, Calif., USA) prior to valve placement. The Chartis method relies on expiratory airflow measurements through the catheter and flow-sensors after a one-way valve has been activated [20]. In cases of airway collapse, the information from the current Chartis system becomes unreliable. We noticed that whenever we saw the endobronchial double-hump 'camelcurve' with our system, we encountered problems with airflow during a Chartis measurement.

We found interesting gas concentration curves when we sampled in front of opening valves. Figure 7 shows sharp rectangular curves with much higher am-

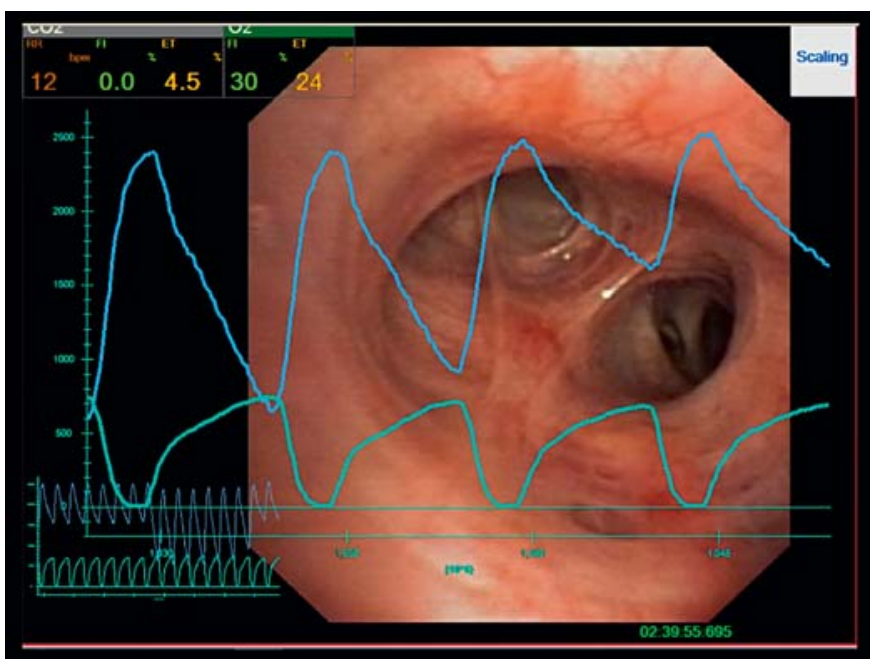

Fig. 5. Between some lobes, there is a remarkable change in the $\mathrm{O}_{2}$ amplitude (upper curve) without any changes in the $\mathrm{CO}_{2}$ waveform (lower curve).

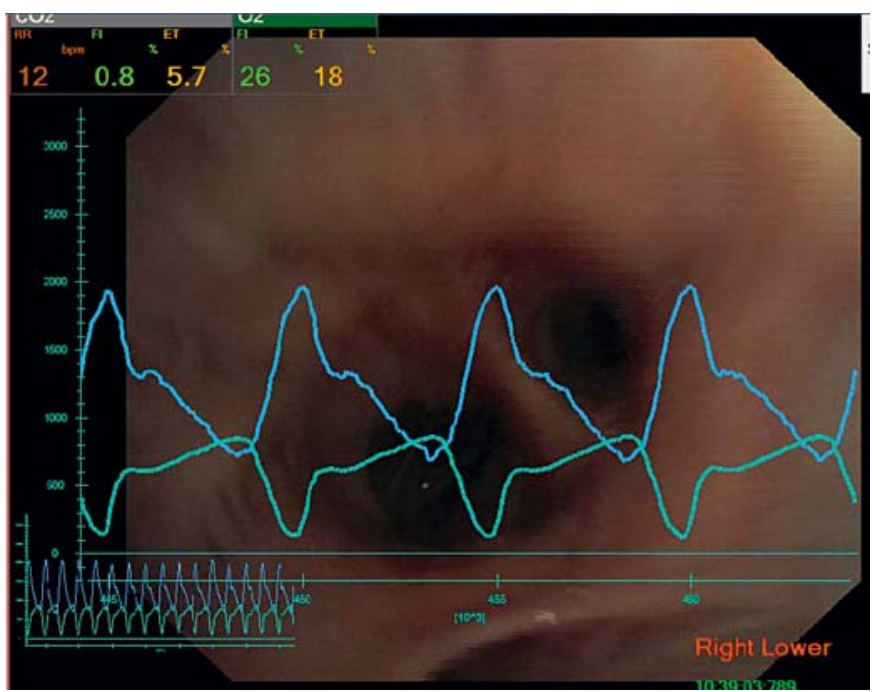

Fig. 6. Visual airway collapses (especially seen in the basal segments), are reflected in the camel-shaped curves of the gas concentration signals. In all patients with these curves, conventional Chartis assessments failed and could not be used for decisionmaking as to whether valves should be placed or not.

plitudes for both gases, most likely indicating venous admixture.

For orientation, we usually sample gases at the entrance to a lobe. In addition, the resolution is high enough to make semiquantitative measurements at the segmental level. In most cases, we have been able to obtain repro- 
Fig. 7. Extreme changes in the $\mathrm{O}_{2}$ (upper) and $\mathrm{CO}_{2}$ (lower) curves, with rectangular shapes and low end-expiratory values, were observed in front of valves.
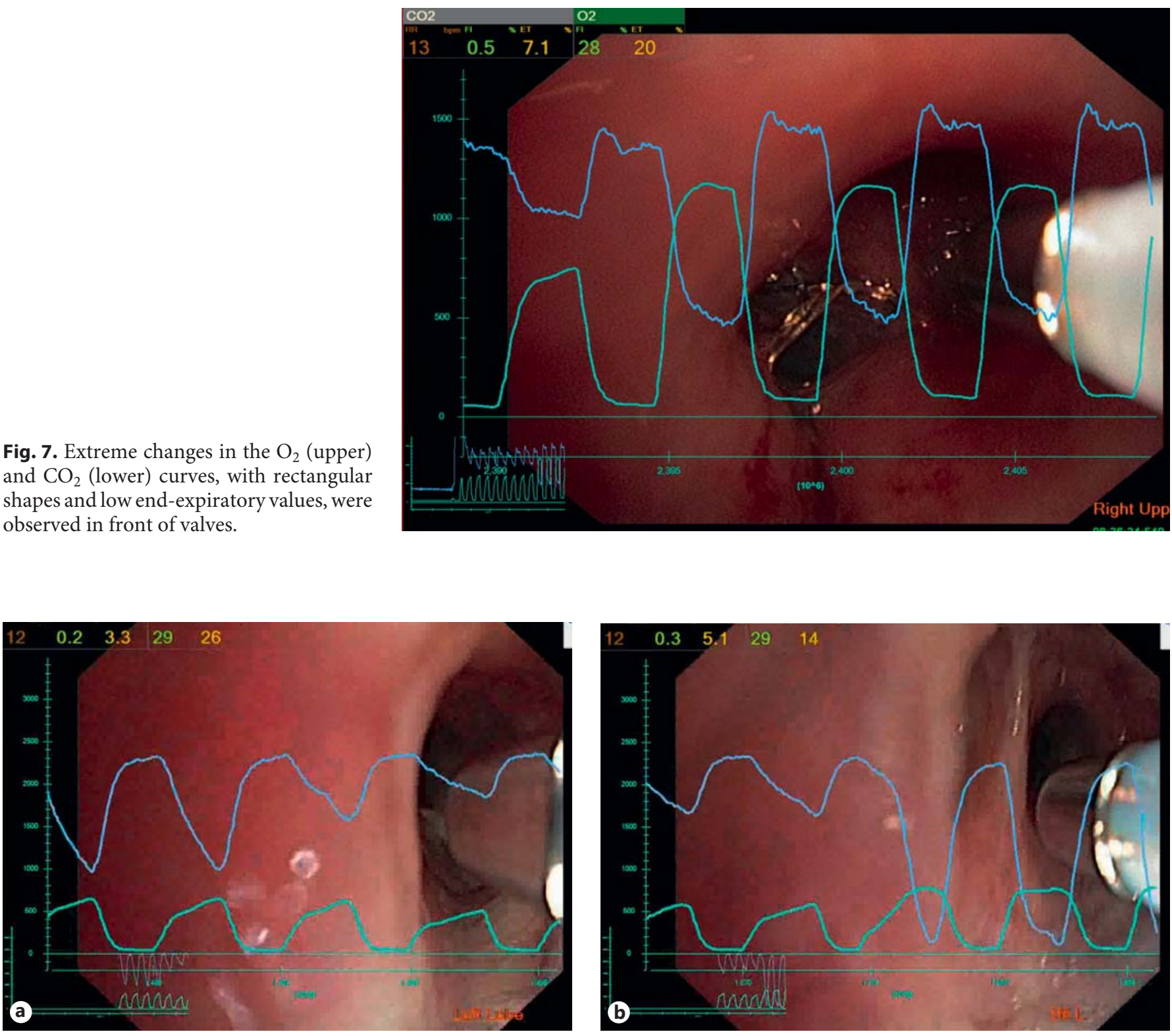

Fig. 8. Measurement resolution is possible to the segmental level. The example shows clear changes in curve shapes and amplitudes if the catheter is moved from the entrance of B9 to B8 (a) and from B8 to B6 (b).

ducible signals by advancing the catheter a few millimeters into the segmental bronchi. Figure 8 demonstrates remarkable differences of amplitudes and shapes in different lower-lobe segments of a patient with advanced bullous emphysema.

Figure 9 shows curves obtained 10 min apart with the catheter placed in the same position. It demonstrates that measurements are reproducible, at least during a single bronchoscopy session.

Endocapnometry and Oximetry Method
The quality of the signals depends on the proper positioning of the sampling catheter. If the catheter is not well centered or the tip touches the mucosa and sucks in mucus, the read-outs cannot be interpreted. It is important to avoid wedge positions. If ventilation of a lobe is significantly impaired by the bronchoscope, no conclusions can be drawn. Figure 10 shows typical examples of distorted signals. 

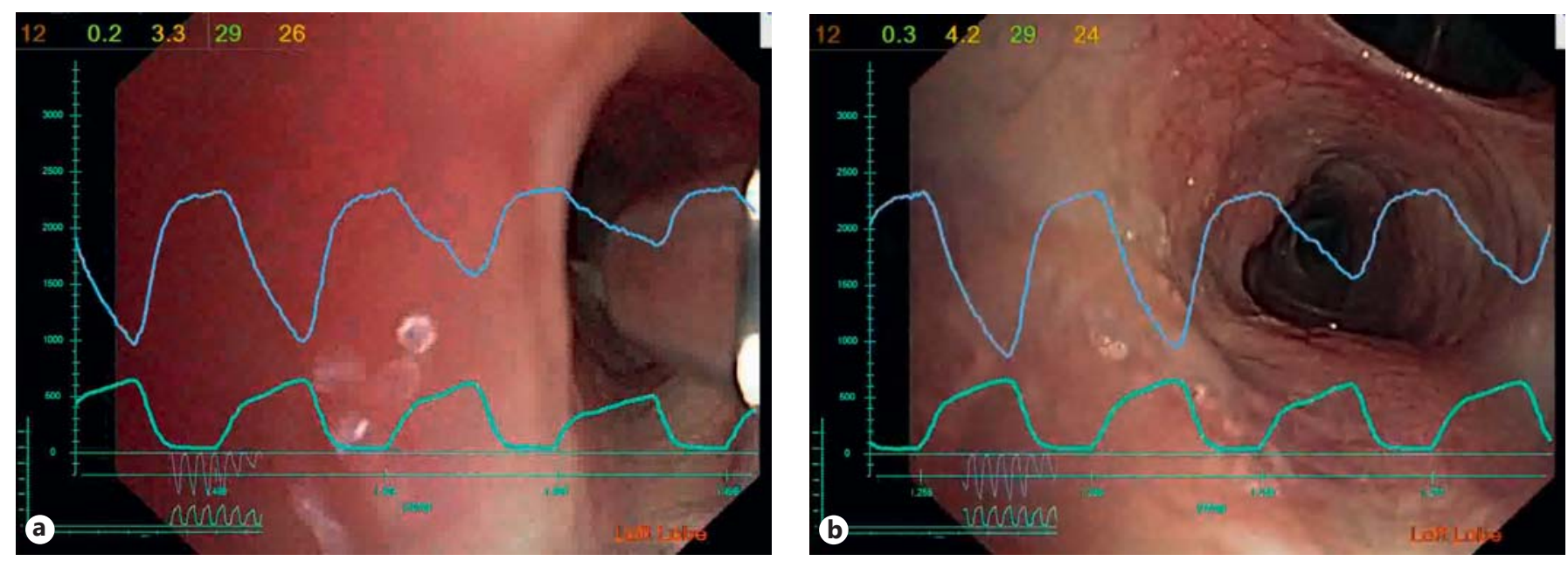

Fig. 9. Signal reproducibility: the reproducibility of measurement is high, at least during the same bronchoscopy session. Measurements were taken 10 min apart in front of B8 (a) and B9 (b). Signal shapes and amplitudes remain similar.
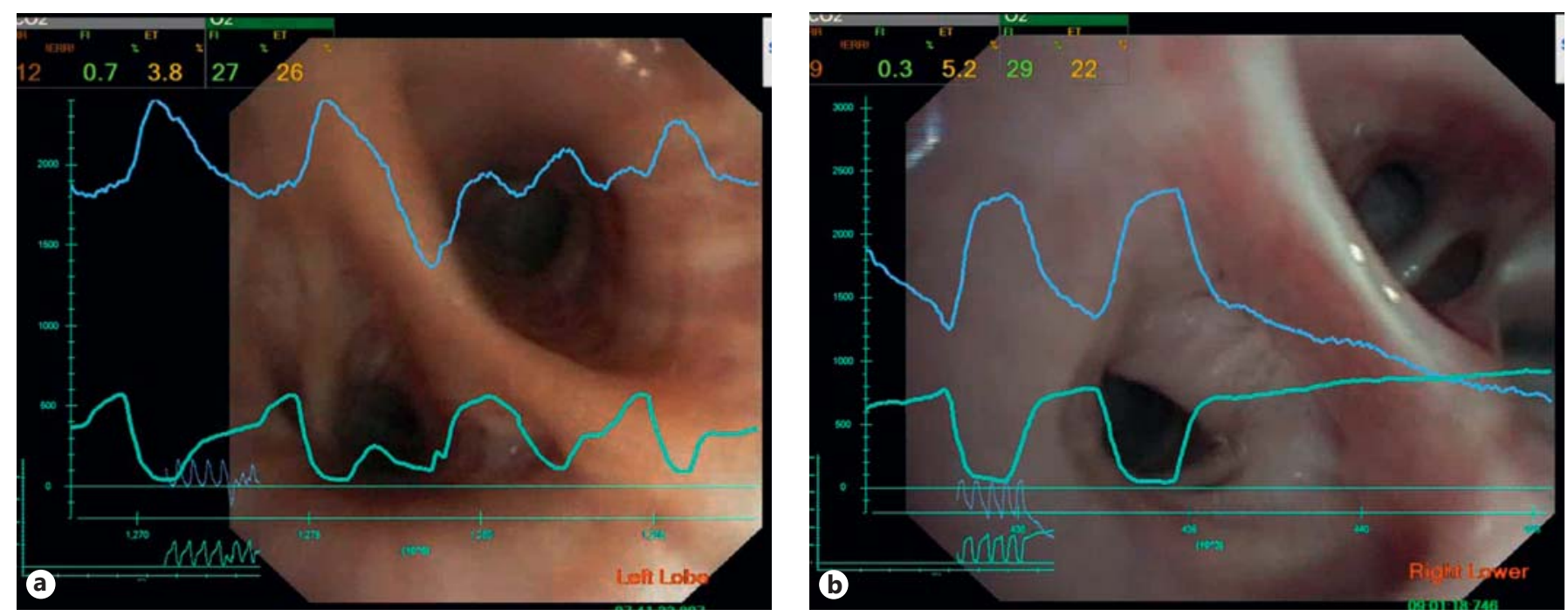

Fig. 10. Signal distortion: touching the mucosa with the tip of the catheter and aspirating mucus (a) or blocking ventilation by advancing the bronchoscope into a wedged position (b) creates artifacts. These are seen on the screen immediately.

\section{Discussion}

Selective bronchoscopic capnography with modified capnometers has been used by other investigators [21]. Although the value of the information obtained by the technique has not been questioned, technical hurdles have prevented its widespread use. In order to improve the feasibility of its clinical application, we have extended the method with selective oximetry and developed a means of presenting the data in real time. The system that we present here is very easy to use. Excessive mucus has to be cleared first and it is important not to touch the mucosa too often. Provided that the bronchoscope is centered in front of a lobe or a segment, we have been able to obtain good signal quality in almost all patients. Of course, the bronchoscope should not block the examined airway. Wedging conditions that impair local ventilation become easily visible as a damped or even flat 
line, as shown in figure 9. With some training, we are able to scan for lobar disturbances in $<5 \mathrm{~min}$. Segmental checking requires more time but it is feasible. We use a 4.2-mm scope for all measurements. Our preferred method of intervention is assisted ventilation of a sedated patient through a laryngeal mask. However, we have used the technique with nasal- and oral-route bronchoscopy in spontaneously breathing patients as well as intubated patients on ventilators. In these patients, it is of interest to observe the effects of changing the ventilator strategy, e.g. the peep level on individual lobes in real time.

There are two types of information available from examination of the signals. First, there are the absolute values of end-inspiratory and end-expiratory gas concentrations. Of course, the inspiratory $\mathrm{O}_{2}$ concentration at the lobar entrance depends on the settings of the ventilator or the amount of supplemental oxygen. We usually set the $\mathrm{FiO}_{2}$ to 0.3 . Almost all patients tolerate an inspiratory oxygen concentration of $30 \%$ when controlled by pulse oximetry. We hypothesize that the amplitude of the $\mathrm{O}_{2}$ signal reflects mostly the local perfusion. The second type of information is the shape and especially the slope of the curves; analogous to normal capnometry, this most likely reflects local ventilation. A triangle capnogram shape in which the plateau is reached very late or not at all indicates physiologically slow spaces. The normal rectangular shape reflects rapid emptying of 'good' parenchyma. These shapes have been described before and methods have been developed to analyze the curves [22-26]. The best of the established methods is that used by Smidt [26], Worth [27] and Yoshimasu et al. [19]. It expresses the slope of the expiratory $\mathrm{CO}_{2}$ concentrations by the percentage of time between 25 and $75 \%$ of the expiratory concentration to the whole time between the lowest value and end-tidal $\mathrm{CO}_{2}$. These calculations can be done easily by software. For the time being, we find that observing the visible shape of the curve when moving from one segment to the next is informative enough. In addition to collecting more data from patients with various diseases, we are also studying the signals in sophisticated ex vivo models in excised lungs. The most valuable feature of the method in a clinical setting is the real-time factor. Instead of analyzing a strip chart afterwards, the endoscopist can receive information immediately and return at any time to check a segment again. The direct overlay of the curves over the endoscopic image helps in interpreting their meanings, as is illustrated by the collapse curves in figure 10. It is reasonable to assume that these shapes are caused by different time constants in the lung compartments.

Endocapnometry and Oximetry Method
There are several limitations to the method presented here. As mentioned above, the curves are influenced by the inspiratory gas concentrations. The level of positive end-expiratory pressure or continuous positive airway pressure can influence the uptakes. The shapes are highly influenced by breathing frequency. We found a rate of 12-15 breaths/min ideal for distinguishing slow and fast spaces. The signals reflect the normal condition of the patient unless the bronchoscope interferes too much with local ventilation. A bronchoscope in or close to a wedge position creates artifacts that can be misleading. The bronchoscope can be positioned at a safe distance and the catheter pushed out of the working channel and placed at the orifice of a segment. However, we found it difficult to avoid touching the mucosa, sucking in mucus and thereby silencing the signal. This problem is not new: it has been addressed by other researchers who have performed endobronchial gas analyses: Hugh-Jones, for example, designed a catheter with a space holder. His instrument had a semi-rigid cone. It would not be too challenging to develop a catheter with a flexible distance holder. For the time being, the real-time display of the curves in combination with the endoimage helps to avoid these technical errors. If, for instance, a flat line is seen, the bronchoscope can immediately be pulled back a few millimeters. We are not aware of a suitable CE-marked device. For legal reasons, we have to use cytology catheters approved for human use. There is certainly room for improvement. A fundamental problem is that the system, due to its sampling method, only displays concentrations. As the true volume cannot be measured with this technique, it is impossible to state how much oxygen is taken up or how much carbon dioxide is really cleared. If a lobe with a high tidal volume and a low oxygen consumption is shut off (e.g. by valves or surgery), it can potentially harm the patient more than the blocking of a lobe with a low oxygen concentration amplitude, if it has a small volume and tidal volume change. Thus, the concentration curves can only be used in a synopsis with imaging techniques. Theoretically, this limitation could be overcome by using a tracer gas technique and a catheter that can be blocked. The product of lobe size and drop in respiratory gas concentrations would be more informative. However, these approaches would only work in the absence of collateral ventilation. The presence of collateral flow limits any dilution method of lobar volume assessment. In addition, blocking a lobe, or even segments, alters gas distribution and computational compensation would probably make it too complicated for daily clinical practice. 
We have tested different capnometers from various manufacturers. The one we currently use is the Phaesin module used in anesthesia monitors. In combination with the catheter, it aspirates at $1.2 \mathrm{ml} / \mathrm{s}$. We made the following estimation: assuming a patient is breathing a tidal volume of $600 \mathrm{ml}$ at a rate of 12 breaths $/ \mathrm{min}$ with an $\mathrm{I}: \mathrm{E}$ ratio of $1: 1$, the result is an expiratory flow of $600 \mathrm{ml}$ in $2.5 \mathrm{~s}$ out of all lobes. Provided that the measured lobe contributes $20 \%$ of the complete ventilation, $48 \mathrm{ml} / \mathrm{s}$ are expired from that lobe while $1.2 \mathrm{ml}$ is aspirated by the device. As $<3 \%$ of the measured gas is aspirated, the influence on the signal is probably negligible. A higher vacuum from the suction pump would shorten the delay time and thereby the examination time, but it might also disturb the signal quality. Another important consideration is the fact that we can only assume that the gas that is collected in front of an orifice does indeed come from this opening. However, if segments are blocked, for example by mucus, the aspirated gas will then come from neighboring segments.

In its current form, we consider our system to be simple, fast and informative. We have used it on a regular basis on emphysema patients who have been treated with endobronchial valves. While the Chartis system for measuring collateral flow helps us in deciding whether a valve should be placed or not, the gas sampling system presented here influences our decision on which lobes should be blocked. We use the CT images, perfusion scans and gas concentration curves to properly select the target lobe for interventional emphysema treatment. We are currently performing observational studies, testing the hypothesis that functional outcomes of emphysema treatments can be improved. If this holds true, this method could be used for decision-making in other interventional procedures, including estimating the degree to which a surgical patient is functionally operable. It might also be useful for newer interventional procedures such as thermoplasty asthma treatment and for optimizing ventilation strategies in patients with inhomogeneous lung diseases. In any case, such a simple method broadens the informative value of bronchoscopy.

\section{Conclusion}

This new method enables gas sampling at the lobe and lung segment levels. The concomitant display of local endocapnometry and endooximetry curves allows for a better identification of target zones for endoscopic emphysema treatments and for the improvement of ventilation strategies for patients on respiratory support.

\section{Acknowledgements}

The authors would like to thank Tuan Nguyen for his expert assistance with designing the system's hardware and software, IB Editorial Services LLC for manuscript editing and Pulmonx Inc. for providing a grant to support the publication of this paper.

\section{References}

1 Flaherty KR, Martinez FJ: The role of computed tomography in emphysema and lung volume reduction surgery; in Lipson $\mathrm{D}$, van Beek E (eds): Functional Lung Imaging. Boca Raton, Taylor \& Francis, 2005, pp 432-451.

2 Olsen GN, Block AJ, Tobias JA: Prediction of postpneumonectomy pulmonary function using quantitative macroaggregate lung scanning. Chest 1974;66:13-16.

3 Loddenkemper R, Gabler A, Göbel D: Criteria of functional operability in patients with bronchogenic carcinoma: preoperative assessment of risk and prediction of postoperative function. Thorac Cardiovasc Surg 1983; 31:334-337.

4 Bolliger CT, Koegelenberg CF, Kendal R: Preoperative assessment for lung cancer surgery. Curr Opin Pulm Med 2005;11:301306

5 Emami K, Chia E, Kadlecek S, MacDuffieWoodburn J, Zhu JL, Pickup S, Blum A, Ishii M, Rizi RR: Regional correlation of emphyse- matous changes in lung function and structure: a comparison between pulmonary function testing and hyperpolarized MRI metrics. J Appl Physiol 2011;110:225-235.

6 Deninger AJ, Mansson S, Petersson JS, Pettersson G, Magnusson P, Svensson J, Fridlund B, Hansson G, Erjefeldt I, Wollmer P, Golman $\mathrm{K}$ : Quantitative measurement of regional lung ventilation using ${ }^{3} \mathrm{He}$ MRI. Magn Reson Med 2002; 48:223-232.

7 Fleming HA, West LR: An appreciation of bronchospirometry as a method of investigation based on 125 cases. Thorax 1954;9:273884.

8 Carlens E: A new flexible double-lumen catheter for bronchospirometry. J Thorac Surg 1949;18:742-746.

9 El Anwar AA: Bronchospirometry and blood gas studies after unilateral tube breathing. Chest 1971;60:347-351.

10 Altenbrunn HJ, Ritzow H, Schleicher H: Study of pulmonary ventilation by means of isotope thoracography following inhalation of Au-198 aerosols and 1st comparison with bronchospirometric results. Radiobiol Radiother (Berl) 1964;5:717-727.

11 Bernadou J, Beck C, Freour P, Blanquet P: Comparative evaluation of pulmonary function by isotope imaging, angiopneumography and differential bronchospirometry. J Nuclear Medicine 1968;10:174-176.

12 Williams SJ, Pierce RJ, Davies NJH, Denisson DM: Methods of studying lobar and segmental function of the lung in man. Br J Dis Chest 1979;73:97-112.

13 Pierce RJ, Brown DJ, Denison DM: Radiographic, scintigraphic, and gas dilution estimates of individual lung and lobar volumes in man. Thorax 1980;35:773-780.

14 Bagg LR, Cox ID: Balloon occlusion of the bronchi at fibreoptic bronchoscopy: application to physiological assessment before lung resection for bronchogenic carcinoma. Tho$\operatorname{rax} 1984 ; 39: 236$. 
15 Corris PA, Kendrick AM, Gibson GJ: Use of bronchoscopic single-breath tests to predict functional results of lobectomy. Thorax 1985; 40:236.

16 Morgan MDL: Physiological aspects of bronchoscopy; in Du Bois RM, Clarke SW (eds): Fiberoptic Bronchoscopy in Diagnosis and Management. London, Gower Medical Publishing, 1987, p 5.20.

17 Hugh-Jones P, West JB: Detection of bronchial and arterial obstruction by continuous gas analysis from individual lobes and segments of the lung. Thorax 1960;15:154-164.

18 Minakata Y, Ikeda T, Nakatani S: Evaluation of expiratory $\mathrm{CO}_{2}$ wave form at lobar level by fibrobronchocapnography. J Jpn Soc Bronchol 1994;16:430-436.
19 Yoshimasu T, Miyoshi S, Maebeya S, Hirai I, Naito Y: Evaluation of effect of lung resection on lobar ventilation and perfusion using intrabronchial capnography. Chest 1996;109: 25-30.

20 Mantri S, Macaraeg C, Shetty S, Aljuri N, Freitag L, Herth F, Eberhardt R, Ernst A: Technical advances: measurement of collateral flow in the lung with a dedicated endobronchial catheter system. J Bronchology Intervent Pulmonol 2009;16:141-144.

21 Füzesi K: Selective capnography through a bronchoscope: a possibility for the functional examination of individual lung segments. Orv Heltil 1980;121:318-395.

22 Visser RJ: Clinical gas analysis on the thermal conductivity. Thesis. Utrecht, University Medical School, 1957.
23 Greve LH: Uneven ventilation. Thesis. Utrecht, University Medical School, 1960.

24 Van Meerten RJ: Expiratory gas concentration curves for examination of uneven distribution of ventilation and perfusion in the lung. Experiments. Respiration 1971;28:167185.

25 Paiva M, Engel LA: The anatomical basis for the sloping alveolar plateau. Respir Physiol 1981;44:325-337.

26 Smidt U: Emphysema as possible explanation for the alteration of expiratory $\mathrm{pO}_{2}$ and $\mathrm{pCO}_{2}$ curves. Bull Eur Physiopathol Respir 1976;12: 605-624.

27 Worth H: Expiratory partial pressure curves in the diagnosis of emphysema. Bull Eur Physiopathol Respir 1986;22:191-199. 